\title{
Jurnal Teknologi Reaktor Nuklir

\section{Radiation Dose Optimization of Breast Cancer with Proton Therapy Method Using Particle and Heavy Ion Transport Code System}

\author{
Milah Fadhilah Kusuma Fasihu ${ }^{1}$, Andang Widi Harto ${ }^{1}$, Isman Mulyadi Triatmoko ${ }^{2}$, Gede Sutrisna Wijaya ${ }^{2}$, and \\ Yohannes Sardjono ${ }^{2}$ \\ ${ }^{1}$ Department of Nuclear Engineering and Physics Engineering, Engineering Faculty, Universitas Gadjah Mada, Jl. Grafika No. 2, Yogyakarta 55281, \\ Indonesia \\ ${ }^{2}$ Center for Accelerator Science and Technology, National Nuclear Energy Agency, Jl Babarsari Mailbox 6101, Yogyakarta 55281, Indonesia
}

\section{ARTICLE INFO}

\section{Article history:}

Received: 30 March 2021

Received in revised form: 28 April 2021

Accepted: 03 Mei 2021

\section{Keywords:}

Breast Cancer

Proton Therapy

Dose Optimization

PHITS

\begin{abstract}
A B S T R A C T
Radiotherapy is one of the cancer treatments conducted by giving a high dose of radiation to the tumor target while minimizing the dose exposed to the healthy organs. One of the available methods is proton therapy. It is usually used in several breast cancer cases while minimizing the damage in the surrounding tissues due to having good precision. In this study, proton therapy in breast cancer will be simulated. This study aims to identify the optimal dose in breast cancer therapy using proton therapy and to identify the dose exposed in the surrounding organs. This study uses simulation based PHITS program to model the geometry and the components of breast cancer and the surrounding organs. The source of radiation is proton with the intensity of $2.62 \times 10^{10}$ proton/s. The variation in beam modelling towards the dose profile of the tumor used is uniform and pencil beam. The proton energy used is $70 \mathrm{MeV}$ up to $120 \mathrm{MeV}$. The result of this study shows that the dose from using pencil beam scanning technique is $50.3997 \mathrm{~Gy}(\mathrm{~W})$ with the total amount of fraction of 25. The dose is below the threshold. Doses in the healthy organs are as follow. The skin received 4.0553 Gy per fraction, the left breast received 0.0011 Gy per fraction, the right breast received 2.6469 Gy per fractions, the right lung received 0.0125 Gy per fraction, the left lung received 0.029 Gy per fraction, the rib received 0.0179 Gy per fraction, and the heart received 0.0077 Gy per fraction.
\end{abstract}

\section{INTRODUCTION}

One of the leading causes of death in the world is breast cancer. In 2020, there were 2.3 million women diagnosed with breast cancer and 685.000 died from it[1], which is about $15 \%$ of all cancer deaths among women. Breast cancer rates are more likely to occur among women in more developed

\footnotetext{
${ }^{*}$ Corresponding author.

E-mail: milah.fadhilah.k@mail.ugm.ac.id

DOI: $10.17146 / \mathrm{tdm} .2021 .23 .2 .6290$
}

regions and have occurred in nearly every region globally.

Generally, breast cancer treatment uses conventional radiotherapy. However, the side effect of this method is considerable for the patient, so other alternatives are needed to reduce the negative impact of the conventional method. Proton therapy is an alternative that can minimize the dose in the healthy tissue around the cancer.

Proton therapy has been widely researched, one of them by Eugen B. Hug titled "Proton Therapy for Primary Breast Cancer." In his research, Hug compared the performance of therapy 
using a proton source with a photon source[2]. The purpose of this research is to determine the optimal dose in breast cancer therapy using proton therapy and to determine the dose exposed to the healthy organs surrounding the cancer cells.

This research focuses on simulating the radiation dose that will be received by mastectomy breast cancer patient with proton therapy. The simulated therapy device is proton therapy using pencil beam scanning (PBS) on the Proteus ${ }^{\circledR O N E}$ system from IBA. The results of this simulation are in the form of doses received by the target tumor and healthy tissue around it.

\section{THEORY}

Proton therapy was first implemented to the human body in 1954. Researchers around the world have been evaluating proton and other therapies over the past four decades. Protons show very good clinical results as it possesses more advantages in radiation oncology. At the moment, proton therapy facilities have spread throughout the hospital almost all over the world[3].

The advantages of proton therapy are its high radiation dose and usability to control and treat cancer with a considerably small risk of damage to healthy tissue and vital organs. These make proton therapy superior to other conventional therapies.

The scheme of how proton therapy works is that the protons are energized at a certain speed to determine the depth of the target in the body, in order to find out the point where the protons will store their maximum energy. When the protons move into the body, slowdown occurs which causes an increase in the interaction of the orbiting electrons[4].

The peak of the electron interaction occurs when the proton approaches the specified target point. Thus, the maximum energy released corresponds to a determined cancer volume. The surrounding healthy tissue received far less injury than at the specified target volume[5].

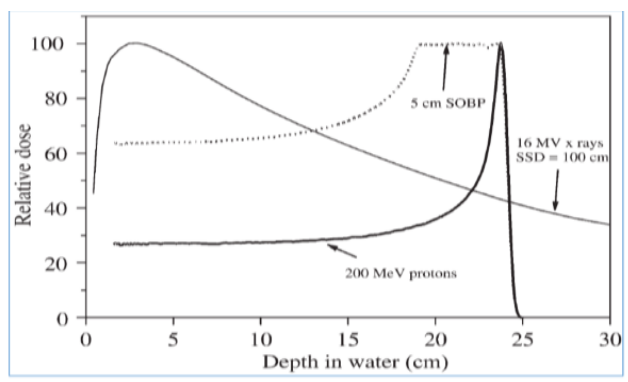

Fig. 1. Bragg curve dose distribution

Proton therapy is highly energy dependent. Most of its energy being stored at the tissue depth determined by the Bragg Peak. The latter is an incident when the peak dose of protons interacts with body tissues before finally disappearing. Dose distribution of proton in matter follows the Bragg curve with a distinct peak, lower entrance dose, and a distal fall-off [6]. Figure 1 show the Bragg curve dose distribution.

Spread Out Bragg Peak (SOBP) is a uniform dosing area that is extended in depth which is formed by the arrangement of the peak dose per depth curve of different energies. SOBP formation starts from selecting the energy range based on the proton range.

In Figure 2, proton therapy has several radiation beam models that can be used in the patient's radiation technique, two of which are[7]:

1. Uniform beam, this beam has a passive scattering system whose scan has a uniform radius of radiation. As a result, healthy tissues around the tumor is at greater risk of being exposed to excessive doses.

2. The pencil beam, which is more advantageous as it has a pencil scanning system. In which, the radiation shot has a different radius to follow the shape of the tumor, which reduces the risk of OAR of being exposed to excessive radiation doses.
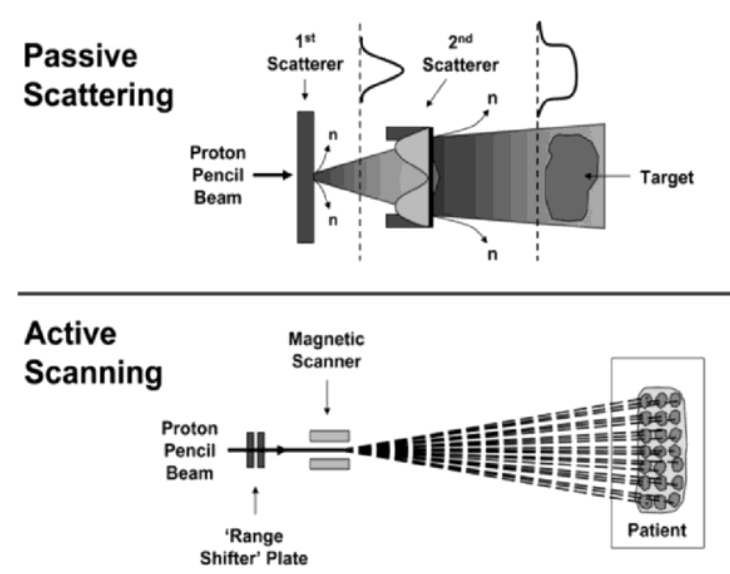

Fig. 2. Beam models of proton delivery in proton therapy[7].

The proton slowdown causes an increase in LET which also results in an increase in relative biological effectiveness (RBE) with the depth of the pure Bragg or SOBP curve. LET is a physical quantity, which indicates the amount of energy transferred to electrons per unit length of paths traversed by charged particles liberated by radioactive decay and/or by radiation interactions in a particular material. The general dimension for LET is kilo electron-volts per micrometer $(\mathrm{keV}$ $\mu \mathrm{m}-1)$. 
$\mathrm{RBE}$ is numerically equivalent to the quality factor of radiation. The quantity of RBE is very different because it represents the ratio of the absorbed dose of the reference radiation in the target volume to the absorbed dose of the desired radiation in the same target, each dose producing the same level of the biological impact of a particular type. Studies indicate the need for different proton RBE values for each patient[8].

LET is a macroscopic dosimetry parameter and does not describe energy deposition in biological targets, but energy deposition per pathway length of particles. However, the use of LET to describe local energy deposition is a valid approximation for proton beams where the number of trace particles traversing the subcellular structure is large[9]. For heavier protons and ions, RBE increases with increasing LET to a maximum and then decreases[10]. For proton, the maximum RBE occurs at very low proton energy which is negligible. Thus, one can safely assume that RBE increases with LET, with the slope depending on the biological endpoint. High LET values in sequential tissue normally located within the target volume may increase the risk of post-proton radiotherapy effects[11].

In the case of breast cancer using proton therapy, the purpose is to provide a total dose of 50.4 Gy (W) to the target and a minimum dose to the healthy organs around the target. The fraction in breast cancer proton therapy is 25 fractions for a total dose of 50.4 Gy (W), where the dose per fraction was 1.8 to 2 Gy[12]. The resulting dose in the simulation is DRBE by multiplying the RBE factor for each particle, 1.1 for protons, 1 for photons and it is assumed 20 for $1 \mathrm{MeV}$ energy neutrons[13].

In radiotherapy, dose fractionation prevents the side effects of radiation by giving healthy tissue time to repair itself[14]. Time of radiation therapy is obtained by dividing the target dose by beam intensity of $2.62 \times 10^{10} \mathrm{proton} / \mathrm{s}$, multiplying factor and weight for each energy level.

Particle and Heavy Ion Transport Code System (PHITS) is a particle transport simulation program. PHITS can simulate most types of particles with energies of up to $1 \mathrm{TeV}$ per nucleon for ions using several nuclear reaction models and data sets[15]. PHITS is commonly used in various research fields such as radiation protection, nuclear medicine and radiological protection.

The source code of PHITS program is written in Fortran language. PHITS simulation can produce a variety of quantities such as heat deposition, line length and production results using the estimator function "calculation" applied. PHITS users can get any information according to their requirements, such as the type of nuclear reaction that is determined according to the computation program writing.

PHITS can be used to calculate the physical dose distribution according to experimental data in the case of proton therapy, these results can indicate that PHITS accurately simulates the proton beamline used for clinical proton therapy[16].

\section{METHODOLOGY}

3.1. Tools

This research was conducted using hardware and software:

1) Laptop with Windows 10 Education 64-bit operating system, Core i5-8250U @1,60 GHz \& 4 GB RAM.

2) PHITS software to simulate particle interactions.

3) Microsoft Excel software to perform data processing.

\subsection{Proton Therapy Specifications}

The beam parameter references to parameters of the Proteus ${ }^{\circledR O N E}$ system used in RSPAD Gatot Soebroto. The proton therapy has the output specifications:

- Particle: Proton

- Beam Energy in patient: 70-230 MeV

- Beam structure: Pulse $1000 \mathrm{~Hz}, 10 \mu \mathrm{s}$, $6 \mathrm{E}+8$ to $1 \mathrm{E}+12$ protons $/ \mathrm{sec}$

- Beam distance to patient: 0.5-32 cm (pencil beam scanning)

- Irradiation time: $\leq 2$ minutes for 2 Gy and a volume of $1 \mathrm{~L}(10 \times 10 \times 10) \mathrm{cm}^{3}$.

\subsection{Parameters}

The parameters used in the simulation in Table 1.

Table 1. Beam parameters in the simulation

\begin{tabular}{llcc}
\hline No & \multicolumn{1}{c}{ Parameters } & Uniform Beam & Pencil Beam \\
\hline 1 & Particle & Proton & Proton \\
2 & Radius (cm) & 2 & $0,1-1,5$ \\
3 & Energy range (MeV) & $70-104$ & $70-106$ \\
4 & Distance to isocenter & 15 & 15 \\
5 & (cm) & 18 & 14 \\
6 & Amount of energy & 1000 & 1000 \\
\hline
\end{tabular}

The distance of the protons and energy can be determined using beam parameters as follows in Table 2. 
Table 2. Beam parameters to determine the depth

\begin{tabular}{ccc}
\hline No & Parameter & Total \\
\hline 1 & Energy range $(\mathrm{MeV})$ & $70-120$ \\
2 & Energy step $(\mathrm{MeV})$ & 2 \\
3 & Beam radius $(\mathrm{cm})$ & 2 \\
4 & Number of particles & 1000 \\
\hline
\end{tabular}

\subsection{Phantom Modeling}

The mathematical phantoms used to model the patient's body geometry are adult female mathematical phantoms from Oak Ridge National Laboratory. The patient's body composition was sourced from the publication of the International Commission on Radiation Units and Measurements (ICRP) Report 4. The result for tissue geometry is depicted in Figure 3.

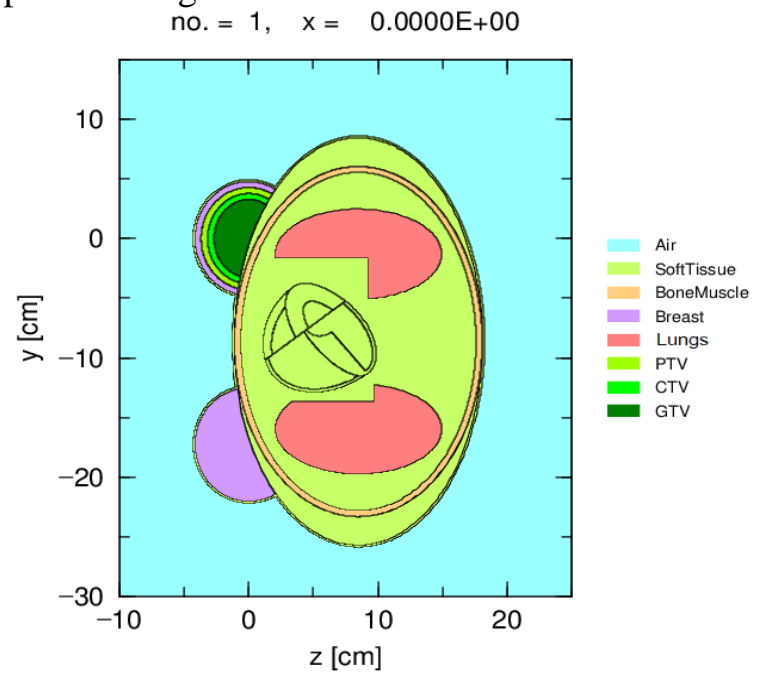

Fig. 3. Anteroposterior modelling looks

As seen above, the type of organs can be classified based on the depth on the Z-axis of the irradiation source. After the geometric shape modeling was completed, a body tissue constituent material was defined, which is made as similar as possible to the tissues of the human body, because in every mass fraction of the tissue constituent elements in each organ passed by radiation has an effect to the obtained dose rate[17].

\subsection{Spread Out Bragg Peak (SOBP)}

SOBP formation starts from selecting the energy range based on the proton range. The energy determined in the simulation is $70-120 \mathrm{MeV}$. Figure 4 is a graph that shows the dose energy of the proton depth.

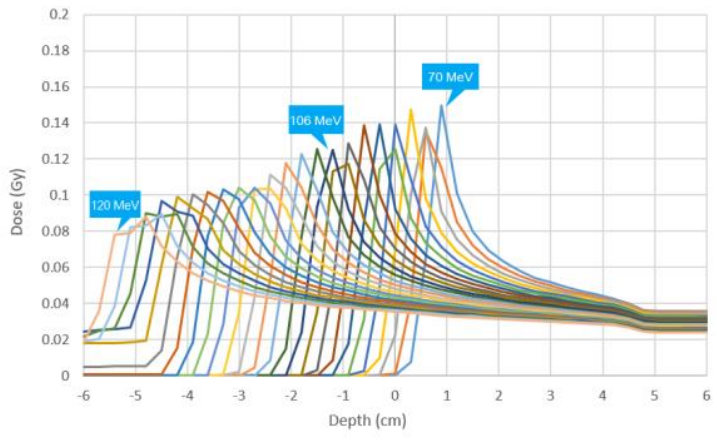

Fig. 4. Depth-dose simulation results for $70-120 \mathrm{MeV}$ proton

In modelling the depth of the tumor that is located $-3-3 \mathrm{~cm}$, the energy chosen in the uniform beam is $70-104 \mathrm{MeV}$, while $70-106 \mathrm{MeV}$ is applied on the pencil beam. Outside that range, energy cannot be entered because the location of the energy peak passes through the body model. SOBP formation is carried out by trial-and-error method to obtain the weight value of each energy. Energy weight has the aim of minimizing the dose, especially the dose in healthy tissue and determining the base dose in the SOBP formation process.

\subsection{Energy Weight}

In this study, variations in beam modelling on the dose profile of the tumor were used. The beams simulated were uniform and pencil beam. These two bundles are simulated to achieve optimal doses.

The direction of beam in the uniform beam is lateral, while for the pencil beam it has two firing directions, that are lateral and left anterior oblique. The choice of lateral and left anterior oblique irradiation on the pencil beam was decided based on the distribution of the dose in the suboptimal lateral firing in the tumor. Therefore, additional shooting direction was still needed to maximize the dose in the tumor.

Furthermore, in the process of forming SOBP, each energy is given weight in the optimal range for building SOBP. Table 3-5 list the weights used for both beams. This process is carried out by trial and error to obtain the energy weight that can cover the entire tumor with an even dose. The highest weight is set at $106 \mathrm{MeV}$ as the foundation of SOBP in the first direction pencil beam scanning (pencil 1) and the highest weight in the second direction pencil beam scanning (pencil 2) with the uniform beam is set at $104 \mathrm{MeV}$. 
Table 3. Energy weights in pencil beam model (pencil 1)

\begin{tabular}{cccc}
\hline Energy $(\mathrm{MeV})$ & Weight & Energy $(\mathrm{MeV})$ & Weight \\
\hline 106 & 1.028 & 86 & 0.0077 \\
104 & 0.15 & 84 & 0.006 \\
102 & 0.12 & 82 & 0.002 \\
100 & 0.085 & 80 & 0.001 \\
98 & 0.03 & - & - \\
96 & 0.024 & - & - \\
94 & 0.02 & - & - \\
92 & 0.01 & - & - \\
90 & 0.0993 & - & - \\
88 & 0.008 & - & - \\
\hline
\end{tabular}

Table 4. Energy weights in uniform beam model (pencil 2)

\begin{tabular}{cccc}
\hline $\begin{array}{c}\text { Energy } \\
(\mathrm{MeV})\end{array}$ & Weight & $\begin{array}{c}\text { Energy } \\
(\mathrm{MeV})\end{array}$ & Weight \\
\hline 104 & 12 & 84 & 1.3 \\
102 & 5 & 82 & 1.2 \\
100 & 3.4 & 80 & 1 \\
98 & 3 & 78 & 0.88 \\
96 & 2.1 & 76 & 0.8 \\
94 & 2 & 74 & 0.7 \\
92 & 2 & 72 & 0.6 \\
90 & 1.6 & 70 & 0.5 \\
88 & 1.4 & - & - \\
86 & 1.4 & - & - \\
\hline
\end{tabular}

Table 5. Dose in organ at risk

\begin{tabular}{cccc}
\hline $\begin{array}{c}\text { Energy } \\
(\mathrm{MeV})\end{array}$ & Weight & $\begin{array}{c}\text { Energy } \\
(\mathrm{MeV})\end{array}$ & Weight \\
\hline 104 & 5 & 84 & 1.4 \\
102 & 4 & 82 & 1.2 \\
100 & 4 & 80 & 1 \\
98 & 3 & 78 & 1.1 \\
96 & 3 & 76 & 1 \\
94 & 3 & 74 & 0.9 \\
92 & 2 & 72 & 0.6 \\
90 & 1.8 & 70 & 0.5 \\
88 & 1.4 & - & - \\
86 & 1.4 & - & - \\
\hline
\end{tabular}

The calculation process using tally. The used tally in this study is [T-Deposit] to calculate the dose. Calculation of irradiation dose along with cancer organ and tissue modelling occurs at the output of PHITS. The results of the simulation will then be processed to produce a calculation of the dose that will be received by the tumor tissue and the healthy tissue around the target. The resulting dose in the simulation is DRBE by multiplying the RBE factor for each particle. The total dose is expressed in Gy (W).

\section{RESULTS AND DISCUSSION}

The difference in Figure 5 and Figure 6 can be seen in the discharge shape of each beam. For Figure 5, it has a beam range that can cover the entire shape of the tumor, due to the pencil beam radiation has a different radius value in each energy so that the beam can adjust the shape of the tumor target. In the uniform beam, the radiation dose shown in Figure 6 has passed PTV and on risk of exposing nearby healthy organs.

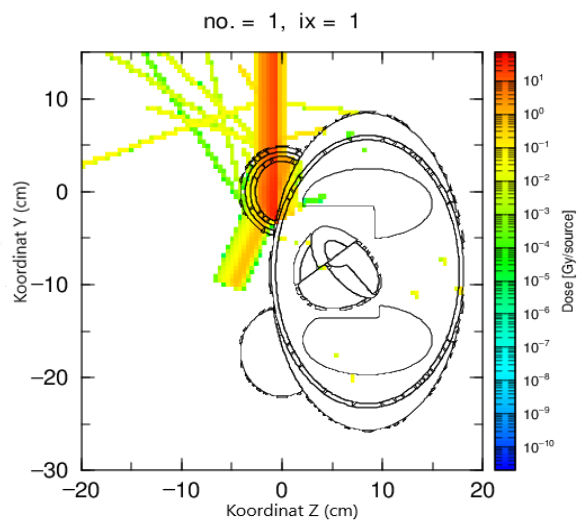

Fig 5. Pencil beam dose profile

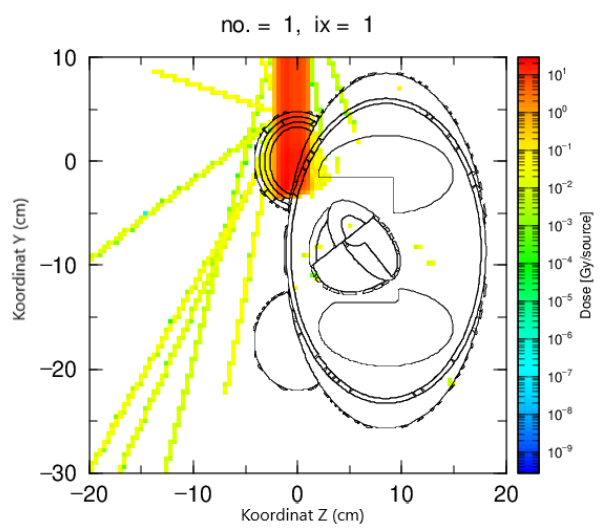

Fig. 6. Uniform beam dose profile

The healthy organs around the tumor are skin, ribs, right and left breast, heart, and lungs. The allowable dose for the skin is $2.2 \mathrm{~Gy} /$ fraction, left breast $0.84 \mathrm{~Gy} /$ fraction, right breast 0.07 Gy/fraction, heart 0.7 Gy/fraction, rib 0.6 Gy/fraction, ipsilateral lung V20 8.11 Gy/fraction and ipsilateral V5 lung $17.13 \mathrm{~Gy} /$ fraction[18].

To obtain the final dose, processing parameter [T-Deposit] was used. The value of the photon and neutron components in therapy has a very small range of values, namely $10^{-1}$ to $10^{-5} \mathrm{~Gy}(\mathrm{~W})$, but still influences the total dose received by the organ. Neutrons and photons are secondary particle components that are released in addition to protons in proton therapy.

Table 6 and Table 7 list the dose distribution in organs and target volumes. The pencil beam gives 50.3997, 4.4475 and $1.2978 \mathrm{~Gy}(\mathrm{~W})$ while the uniform beam gives 52.5244, 9.55139, and 2.37621 Gy (W) in GTV, CTV and PTV. 
Table 6. Pencil beam organ dose

\begin{tabular}{|c|c|c|c|c|}
\hline Organ & $\begin{array}{c}\text { Proton } \\
\text { Dose } \\
\text { (Gy RBE) }\end{array}$ & $\begin{array}{c}\text { Photon } \\
\text { Dose } \\
\text { (Gy RBE) }\end{array}$ & $\begin{array}{l}\text { Neutron } \\
\text { Dose } \\
\text { (Gy RBE) }\end{array}$ & $\begin{array}{c}\text { Total Dose } \\
(\text { Gy }(W))\end{array}$ \\
\hline PTV & 1.281 & 0.00053 & 0.0167 & 1.2978 \\
\hline CTV & 4.395 & 0.0015 & 0.0507 & 4.4475 \\
\hline GTV & 50.221 & 0.00464 & 0.1741 & 50.3997 \\
\hline Right Breast & 2.6079 & 0.00115 & 0.03788 & 2.6469 \\
\hline Left Breast & 0.0001 & 0.00001 & 0.00107 & 0.0011 \\
\hline Right Lung & 0.0002 & 0.00024 & 0.01206 & 0.0125 \\
\hline Left Lung & 0 & 0.00004 & 0.00282 & 0.0029 \\
\hline Skin & 4.0542 & 0.00003 & 0.00103 & 4.05532 \\
\hline Rib & 0.014 & 0.00013 & 0.00389 & 0.0179 \\
\hline Heart & 0.00006 & 0.0002 & 0.0074 & 0.0077 \\
\hline Trunk & 0.00255 & 0.00004 & 0.00153 & 0.0041 \\
\hline \multicolumn{5}{|c|}{ Table 7. Uniform beam organ dose } \\
\hline Organ & $\begin{array}{c}\text { Proton } \\
\text { Dose } \\
\text { (Gy RBE) } \\
\end{array}$ & $\begin{array}{c}\text { Photon } \\
\text { Dose } \\
\text { (Gy RBE) } \\
\end{array}$ & $\begin{array}{c}\text { Neutron } \\
\text { Dose } \\
\text { (Gy RBE) }\end{array}$ & $\begin{array}{c}\text { Total Dose } \\
(\text { Gy }(\mathrm{W}))\end{array}$ \\
\hline PTV & 2.17547 & 0.00074 & 0.2 & 2.37621 \\
\hline CTV & 8.79989 & 0.0021 & 0.7494 & 9.55139 \\
\hline GTV & 50.38 & 0.0056 & 2.1388 & 52.5244 \\
\hline Right Breast & 3.74979 & 0.0017 & 0.4678 & 4.21929 \\
\hline Left Breast & 0 & 0.00003 & 0.1042 & 0.10423 \\
\hline Right Lung & 0.00101 & 0.0004 & 0.112 & 0.11341 \\
\hline Left Lung & 0 & 0.00004 & 0.0442 & 0.04424 \\
\hline Skin & 4.5363 & 0.00006 & 0.0164 & 4.55276 \\
\hline Rib & 0.4085 & 0.0003 & 0.106 & 0.5148 \\
\hline Heart & $7.98 \mathrm{E}-05$ & 0.0003 & 0.1938 & 0.19418 \\
\hline Trunk & 0.035 & 0.00006 & 0.0236 & 0.05866 \\
\hline
\end{tabular}

The results of both files are relatively the same. The data in Table 6 and Table 7 show that the dosage in pencil beam in the tissues around the target is lower than the uniform beam, which is relatively higher due to the wider distal and lateral profile. This is because the pencil beam has a radius variation, which can limit the proton exposure. Therefore, pencil beam is the more optimal selection in this study.

Table 8 shows the value of the dose received by organs at risk per fraction and the total fraction. In this study, it is indicated that the dose received by organs around the tumor tissue are below the allowable limit.

Table 8. Total dose results on organs at risk

\begin{tabular}{|c|c|c|c|c|c|}
\hline \multirow[t]{2}{*}{ Organ at risk } & \multicolumn{2}{|c|}{ Pencil beam } & \multicolumn{2}{|c|}{ Uniform beam } & \multirow{2}{*}{$\begin{array}{c}\text { Allowable } \\
\text { limit } \\
\text { (Gy/Fraction) }\end{array}$} \\
\hline & $\begin{array}{l}\text { Fraction } \\
(\mathrm{Gy}(\mathrm{W}))\end{array}$ & $\begin{array}{c}\text { Total } \\
(\mathrm{Gy}(\mathrm{W}))\end{array}$ & $\begin{array}{l}\text { Fraction } \\
(\mathrm{Gy}(\mathrm{W}))\end{array}$ & $\begin{array}{c}\text { Total } \\
(\mathrm{Gy}(\mathrm{W}))\end{array}$ & \\
\hline Skin & 0.1622 & 4.0553 & 0.182110 & 4.55276 & 2.2 \\
\hline $\mathrm{R}$ Breast & 0.1059 & 2.6469 & 0.168771 & 4.21929 & 0.07 \\
\hline L Breast & 0.0000 & 0.0011 & 0.004169 & 0.10423 & 0.84 \\
\hline Hearts & 0.0003 & 0.0077 & 0.007767 & 0.19418 & 0.7 \\
\hline Rib & 0.0007 & 0.0179 & 0.020592 & 0.5148 & 0.6 \\
\hline R Lung & 0.0005 & 0.0125 & 0.004536 & 0.11341 & 8.11 \\
\hline L Lung & 0.0012 & 0.029 & 0.001769 & 0.04424 & 17.13 \\
\hline
\end{tabular}

\section{CONCLUSION}

In this study, two radiation techniques were simulated in proton therapy for breast cancer, namely pencil beam scanning and passive scattering (uniform beam). Based on the research results, the pencil beam scanning technique has a total dose value that is closer to 50.4 Gy than the passive scattering technique. The results of the dose of using pencil beam scattering for breast cancer using proton therapy are 50.3997 Gy (W) with a total fraction of 25 and the received dose of healthy tissue is still below the allowable dose where the skin gets 4.0553 Gy (W) per fraction, breast left $0.0011 \mathrm{~Gy}(\mathrm{~W})$ per fraction, right breast $2.6469 \mathrm{~Gy}$ (W), right lung 0.0125 Gy (W) per fraction, left lung $0.029 \mathrm{~Gy}(\mathrm{~W})$ per fraction, rib $0.0179 \mathrm{~Gy}(\mathrm{~W})$ per fraction, and heart $0.0077 \mathrm{~Gy}(\mathrm{~W})$ per fraction.

\section{ACKNOWLEDGMENT}

The authors acknowledge LPDP Ministry of Finance, Department of Nuclear Engineering and Physics Engineering, Engineering Faculty, Universitas Gadjah Mada, and Center for Accelerator Science and Technology, National Nuclear Energy Agency (BATAN).

\section{AUTHOR CONTRIBUTORSHIP}

Milah Fadhilah Kusuma Fasihu is the main contributor of this paper. Yohannes Sardjono and Andang Widi Harto supervised throughout this study. All authors read and approved the final version of the paper.

\section{REFERENCE}

1. World Health Organization, Breast Cancer. WHO. 2021. https://www.who.int/newsroom/fact-sheets/detail/breast-cancer.

2. Hug E.B. Proton Therapy for Primary Breast Cancer. Breast Care. 2018. 13(3): 168-172.

3. Newhauser W.D., Zhang R. The Physics of Proton Therapy. Physics in Medicine and Biology. 2015. 60: 155-209.

4. Dinesh Mayani D. Proton Therapy for Cancer Treatment. J. Oncol. Pharm. Pract. 2011. 17(3): 186-190.

5. Otero J., Felis I., Herrero A., Merchán J.A., Ardid M. Bragg Peak Localization with Piezoelectric Sensors for Proton Therapy Treatment. Sensors (Switzerland). 2020. 20(10): 2987.

6. Chuong M., Kaiser A., Molitoris J., Romero A.M., Apisarnthanarax S. Proton Beam Therapy for Liver Cancers. Journal of 
Gastrointestinal Oncology. 2020. 11(1): 157165.

7. Leroy R., Benahmed N., Hulstaert F., Mambourg F., Fairon N., Van Eycken E., et al. Hadron Therapy in Children - an Update of the Scientific Evidence for 15 Paediatric Cancers. 2015. KCE Report 235.

8. Chen Y., Grassberger C., Li J., Hong T.S., Paganetti H. Impact of Potentially Variable RBE in Liver Proton Therapy. Phys. Med. Biol. 2018. 63(19): 195001.

9. Paganetti H. Relative Biological Effectiveness (RBE) Values for Proton Beam Therapy. Variations as a Function of Biological Endpoint, Dose, and Linear Energy Transfer. Physics in Medicine and Biology. 2014. 59(22): R419.

10. Choi J., Kang J.O. Basics of Particle Therapy II: Relative Biological Effectiveness. Radiation Oncology Journal. 2012. 30(1): 113.

11. Unkelbach J., Botas P., Giantsoudi D., Gorissen B.L., Paganetti H. Reoptimization of Intensity Modulated Proton Therapy Plans Based on Linear Energy Transfer. Int. J. Radiat. Oncol. Biol. Phys. 2016. 96(5): 10971106

12. Bekelman J.E., Lu H., Pugh S., Baker K., Berg C.D., De Gonzalez A.B., et al. Pragmatic Randomised Clinical Trial of Proton Versus Photon Therapy for Patients with Nonmetastatic Breast Cancer: The Radiotherapy Comparative Effectiveness (RadComp) Consortium Trial Protocol. BMJ Open. 2019. 9(10): e025556.

13. Lechner A. Particle interactions with matter. in: CERN Yellow Reports: School Proceedings. 2018.

14. Marshall T.I., Chaudhary P., Michaelidesová A., Vachelová J., Davídková M., Vondráček V., et al. Investigating the Implications of a Variable RBE on Proton Dose Fractionation Across a Clinical Pencil Beam Scanned Spread-Out Bragg Peak. Int. J. Radiat. Oncol. Biol. Phys. 2016. 95(1): 70-77.

15. Sato T., Iwamoto Y., Hashimoto S., Ogawa T., Furuta T., Abe S. ichiro, et al. Features of Particle and Heavy Ion Transport code System (PHITS) version 3.02. J. Nucl. Sci. Technol. 2018. 55(6): 684-690.

16. Takada K., Sato T., Kumada H., Koketsu J., Takei H., Sakurai H., et al. Validation of the Physical and RBE-weighted Dose Estimator Based on PHITS Coupled with a Microdosimetric Kinetic Model for Proton Therapy. J. Radiat. Res. 2018. 59(1): 91-99.
17. Siwi D.B. Analisis Dosis di Organ Kritis pada Terapi Globlastoma dengan Boron Neutron Capture Therapy Menggunakan Metode Simulasi MCNP5. Thesis, UGM. 2013.

18. Hutnik M., Składowski K., Wygoda A., Rutkowski T., Pilecki B. Tolerance Doses of Critical Organs in Patients with Head and Neck Cancer Treated with Radiation Therapy. Nowotwory. 2013. 63(1): 35-47. 